\title{
Motoric impairment versus iron deposition gradient in the subthalamic nucleus in Parkinson's disease
}

\author{
Weiyuan Huang, MD, ${ }^{1,7}$ Richard Ogbuji, MD, ${ }^{2}$ Liangdong Zhou, PhD, ${ }^{1}$ Lingfei Guo, MD, ${ }^{1}$ \\ Yi Wang, PhD, ${ }^{1,6}$ and Brian H. Kopell, MD2-5 \\ 1Department of Radiology, Weill Medical College of Cornell University, New York; Departments of ${ }^{2}$ Neurosurgery, ${ }^{3}$ Neurology, \\ ${ }^{4}$ Psychiatry, and ${ }^{5}$ Neuroscience, Icahn School of Medicine at Mount Sinai, New York; ${ }^{6}$ Meinig School of Biomedical Engineering, \\ Cornell University, Ithaca, New York; and 'Department of Radiology, Hainan General Hospital (Affiliated Hainan Hospital of \\ Hainan Medical University), Haikou City, People's Republic of China
}

OBJECTIVE The objective of this study was to investigate the correlation between the quantitative susceptibility mapping (QSM) signal gradient of the subthalamic nucleus (STN) and motor impairment in patients with Parkinson's disease (PD).

METHODS All PD patients who had undergone QSM MRI for presurgical deep brain stimulation (DBS) planning were eligible for inclusion in this study. The entire STN and its three functional subdivisions, as well as the adjacent white matter (WM), were segmented and measured. The QSM value difference between the entire STN and adjacent WM (STNWM), between the limbic and associative regions of the STN $(L-A)$, and between the associative and motor regions of the STN (A-M) were obtained as measures of gradient and were input into an unsupervised k-means clustering algorithm to automatically categorize the overall boundary distinctness between the STN and adjacent WM and between STN subdivisions (gradient blur [GB] and gradient sharp [GS] groups). Statistical tests were performed to compare clinical and image measurements for discrimination between GB and GS groups.

RESULTS Of the 39 study patients, 19 were categorized into the GB group and 20 into the GS group, based on quantitative cluster analysis. The GB group had a significantly higher presurgical off-medication Unified Parkinson's Disease Rating Scale Part III score (51.289 \pm 20.741$)$ than the GS group ( $38.5 \pm 16.028 ; p=0.037)$. The GB group had significantly higher QSM values for the STN and its three subdivisions and adjacent WM than those for the GS group $(p<$ 0.01). The GB group also demonstrated a significantly higher STN-WM gradient in the right STN $(p=0.01)$. The GB group demonstrated a significantly lower L-A gradient in both the left and the right STN $(p<0.02)$.

CONCLUSIONS Advancing PD with more severe motor impairment leads to more iron deposition in the STN and adjacent WM, as shown in the QSM signal. Loss of the STN inner QSM signal gradient should be considered as an image marker for more severe motor impairment in PD patients.

https://thejns.org/doi/abs/10.3171/2020.5.JNS201163

KEYWORDS subthalamic nucleus; quantitative susceptibility mapping; deep brain stimulation; Parkinson's disease; functional neurosurgery

$\mathrm{T}$ HE subthalamic nucleus (STN) is a glutamatergic nucleus situated in the diencephalon that is critical for the regulation of motor function through both the direct and indirect motor pathways connected to the basal ganglia and also plays a role in limbic and associative processing. ${ }^{1}$ A hallmark of Parkinson's disease (PD) is an increase in and abnormal synchronization of STN neuronal activity; thus, the STN is a primary target for deep brain stimulation (DBS) therapy., ${ }^{2,3}$ DBS of the STN has been shown to provide significant benefit to patients with PD, particularly with regard to motor outcomes. ${ }^{4}$ Additionally, other studies have suggested that STN DBS may provide superior improvement in off-period bradykinesia in patients with PD.

Precise placement of the stimulation electrode is of the utmost importance to the success of DBS surgery. MRI is used to locate and navigate the STN during surgical procedures because of the high tissue contrast of the imaging

ABBREVIATIONS A-M = QSM value differences between associative and motor regions of STN; DBS = deep brain stimulation; GB = gradient blur; GRE = gradient echo; GS = gradient sharp; L-A = QSM value differences between limbic and associative regions of STN; PD = Parkinson's disease; $Q S M=$ quantitative susceptibility mapping; RN = red nucleus; SN = substantia nigra; STN = subthalamic nucleus; STN-WM = QSM value differences between entire STN and adjacent WM; UPDRS-III = Unified Parkinson's Disease Rating Scale Part III; WM = white matter.

SUBMITTED April 9, 2020. ACCEPTED May 12, 2020.

INCLUDE WHEN CITING Published online August 7, 2020; DOI: 10.3171/2020.5.JNS201163. 
modality. Quantitative susceptibility mapping (QSM), ${ }^{6}$ generated from the multiple-echo gradient echo (GRE) sequence, has better contrast and delineation of the STN than conventional MRI by exploiting variability in tissue iron-containing neuromelanin deposition. ${ }^{7,8}$ Histological and QSM studies ${ }^{9}$ have revealed an inhomogeneous iron distribution within the STN in healthy volunteers, which has been related to three functional subdivisions of the STN-limbic, associative, and motor regions. ${ }^{3}$ The limbic, associative, and motor regions reside in the anatomical anterior, mid, and posterior portions of the nucleus, respectively. ${ }^{10-12}$ However, iron deposition in certain patients with PD appears well laminated and divided along the length of the nucleus; in other patients, the boundary between functional subdivisions is poorly defined, leading to the loss of an iron signal gradient in the STN. There are no published studies to validate the relationship between the iron signal gradient of the STN on QSM MR images and the disease status of PD. In this study, we aimed to investigate the relationship between the QSM signal gradient of the STN and motor impairment in patients with PD.

\section{Methods \\ Patient Population}

All patients with PD who had undergone MRI for presurgical DBS planning at our hospital from October 2018 to August 2019 were eligible for inclusion in this retrospective study, which was approved by our IRBs at Mount Sinai and Cornell. The PD diagnosis was made by experienced neurologists. Exclusion criteria consisted of 1) a history of other neurological or psychiatric disorders or brain trauma, 2) previous surgery or radiotherapy in the STN region, and 3) MRI contraindications (e.g., artificial cochlea, cardiac pacemaker implantation) or DBS contraindications. The Unified Parkinson's Disease Rating Scale Part III (UPDRS-III) motor examination scores in the on-medication and off-medication states were obtained to assess motor impairment preoperatively. The motor subscale consists of 14 items (including assessments of facial expression, speech, tremor, rigidity, bradykinesia, gait, and posture), with score totals ranging from 0 to 100 and higher scores indicating greater impairment. ${ }^{13}$ The score was determined by a trained neurologist's examination of the patient.

\section{MRI Acquisition}

All subjects were scanned using a 3-T MRI system (Discovery MR750 scanner, GE Healthcare) with an 8-channel head coil. QSM images were acquired using a 3D multiecho GRE sequence with the following parameters: TR $44.1 \mathrm{msec}$, TE $3.8 \mathrm{msec}$, echo number 10, bandwidth $\pm 62.5 \mathrm{kHz}$, number of excitations 0.75 , FOV $256 \times$ $256 \mathrm{~mm}^{2}$, acquisition matrix $320 \times 320 \times 180$, voxel size $0.5 \times 0.5 \times 0.5 \mathrm{~mm}^{3}$, and scan time 13 minutes. All images were acquired while the patient was under full general endotracheal anesthesia in order to eliminate motion to ensure image quality.

\section{Images Postprocessing}

QSM of the whole brain was reconstructed using the following pipeline: image preprocessing included field estimation and spatial unwrapping from multiecho GRE data, background field removal by the projection onto dipole fields (PDF) method, and QSM reconstruction from the local field by a morphology-enabled dipole inversion with zero reference (MEDI+0) ${ }_{\cdot}^{14-16}$ In order to make a zero reference inside CSF during QSM reconstruction, the CSF area was first automatically segmented using $\mathrm{R}_{2}$ * information from multiecho magnitude images. A regularization term was then applied to achieve homogeneous susceptibility values in the region inside CSF.

\section{Image Analysis: Radiological Visual Analysis}

Two experienced radiologists (W.H. and L.G.), who were blinded to the clinical information, independently evaluated the boundary gradient and subdivision gradient of the STN on QSM images. Localization of the STN was based on a combination of relationships to the red nucleus $(\mathrm{RN})$ and the substantia nigra (SN). First, the central slice of the RN and SN on coronal QSM images was found. Then, the STN, which is oriented at an angle against the anteromedial side of the SN, was localized. The STN was then localized on coregistered axial and sagittal planes.

Each STN (left and right) was scored according to its boundary gradient and subdivision gradient using a 2 -point scale. The STN boundary gradient was radiologically scored as follows: 1 if the boundary between the STN and adjacent SN/white matter (WM) was clearly defined (sharp), and 0 if the boundary between the STN and adjacent SN/WM was poorly defined (blur). The STN subdivision gradient was radiologically scored as follows: 1 if the subdivision gradient distinctly existed among the three functional subdivisions (limbic, associative, and motor), and 0 if the subdivision gradient was indistinct or nonexistent (blur). The final score was calculated as the average of the two observers. Then, all these radiological scores for both STNs were added together for each subject. If a patient had a total score of 4 , the patient was categorized into the gradient sharp (GS) group; otherwise, patients with a total score of 3 or lower were categorized into the gradient blur (GB) group.

\section{Image Analysis: Quantitative Cluster Analysis}

The STN and adjacent WM (a 0.5- to 1-mm-thick band immediately surrounding the STN) were manually segmented in ITK-SNAP software (version 3.4.0, http://www. itksnap.org). ${ }^{17}$ The STN segmentations were further subdivided into three parts equally, representing the limbic, associative, and motor function regions, from ventromedial to dorsolateral (Fig. 1). Although the exact subdivision zones are likely of different sizes, equal tripartite division of the STN functional zones is a currently accepted conventional approximation. The QSM values for the entire STN, adjacent WM, and limbic, associative, and motor regions of the STN were measured. The QSM value differences between the entire STN and the adjacent WM (STN-WM), between the limbic and associative regions of the STN (L-A), and between the associative and motor regions of the STN (A-M) were calculated.

Then GS and GB categorization was generated in the 


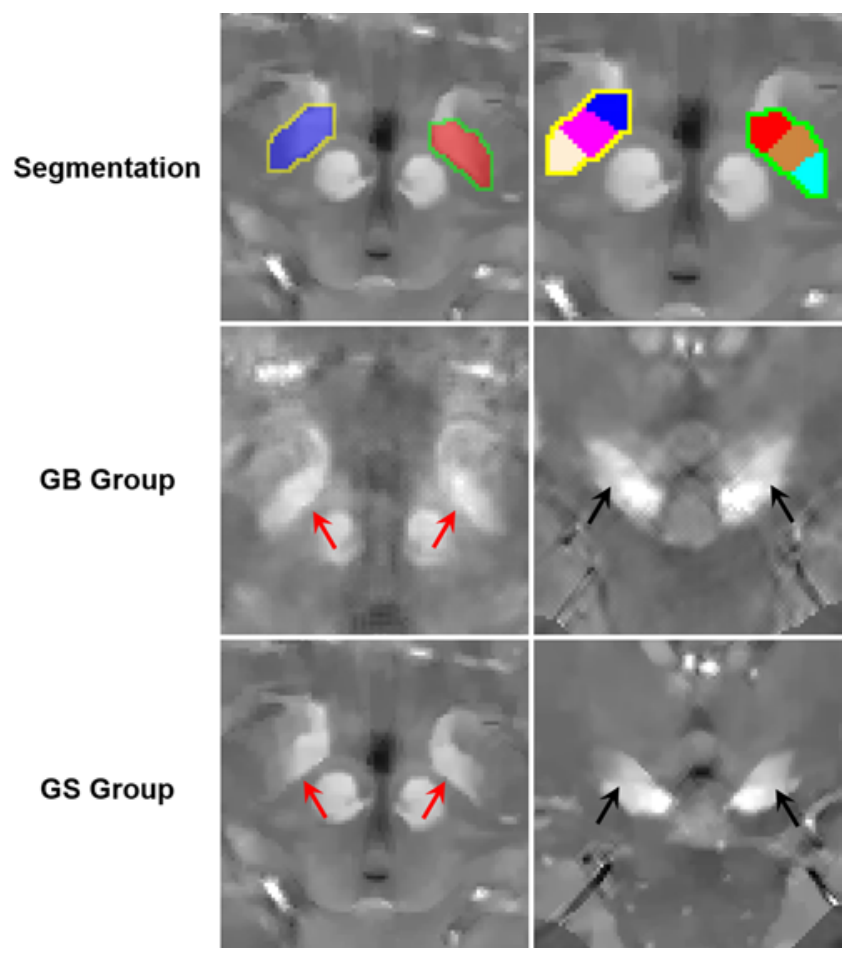

FIG. 1. STNs and adjacent WM were manually segmented. The STN segmentations were further subdivided into three parts equally, representing the limbic, associative, and motor functional regions. The GS group QSM showed a clear boundary and subdivision signal gradient. However, the GB group showed a blurred boundary and loss of subdivision signal gradient within the STN. Red arrows indicate the boundary between the STN and adjacent WM; black arrows, the boundary between the STN and SN. Figure is available in color online only.

following manner. Region-averaged QSM and QSM gradient data were assembled as a $39 \times 16$ data matrix, each column corresponding to a subregion's mean QSM or the QSM difference between two adjacent subregions, which was then inputted into the unsupervised k-means clustering algorithm using the Spearman correlation distance with the number of clusters set to 2 and the iteration number set to $100 .^{18}$ The cluster with a larger average QSM gradient was identified as the GS group.

\section{Statistical Analysis}

Statistical analysis was performed using MATLAB routines (2019 version, MathWorks). The QSM values for the entire STN, the adjacent WM, the limbic, associative, and motor regions of the STN, the STN boundary gradient (STN-WM QSM value), and the STN subdivision gradient (L-A QSM value and A-M QSM value); the radiological visual score; the UPDRS-III score; and disease duration were assessed in the two groups using an independentsamples t-test. Patient sex was assessed in the two groups using a chi-square test. The interrater reliability of the two neuroradiologists was evaluated using Cohen's kappa test. The Spearman correlation between QSM gradient and UPDRS-III score was also evaluated. A p value $<0.05$ was considered statistically significant.

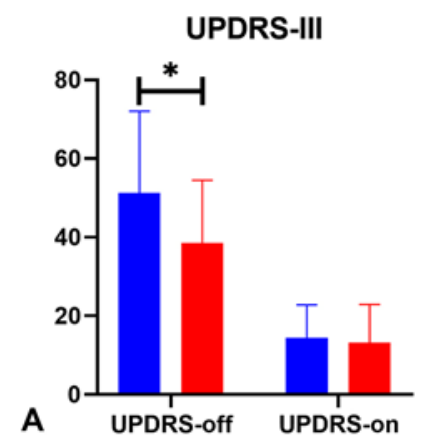

Left STN Radiological Score

Right STN Radiological Score
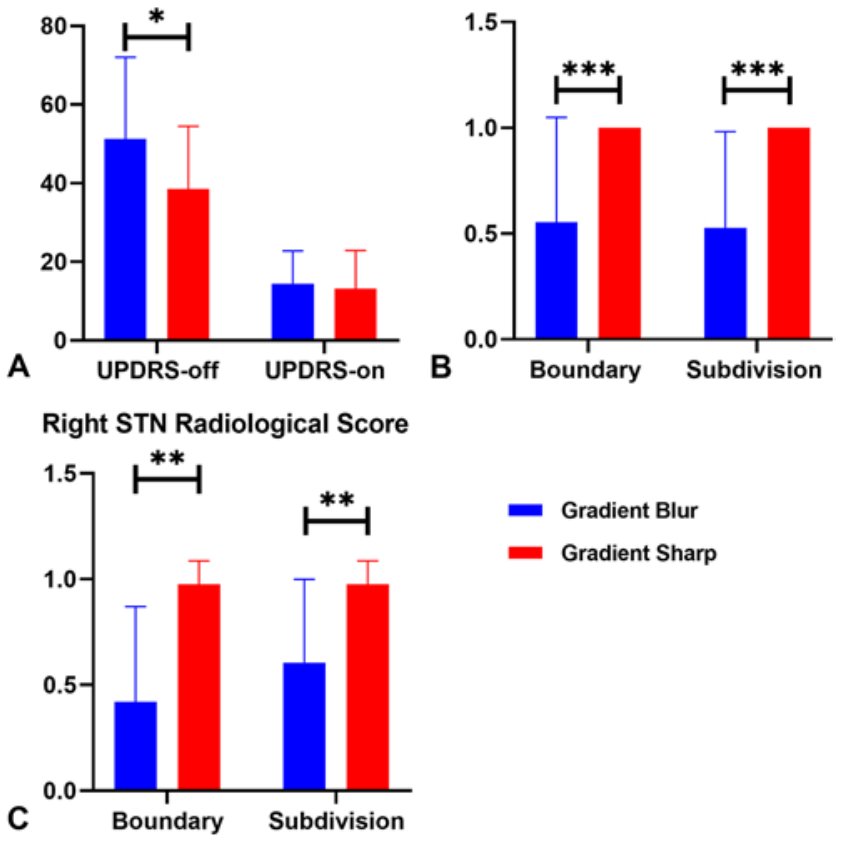

FIG. 2. The UPDRS-III score (A) and radiological score (B and C) distributions for the two groups. The GB group had a significantly higher presurgical off-medication UPDRS-III score and lower radiological score than the GS group. ${ }^{*} p<0.05,{ }^{* *} p<0.01$, and ${ }^{* * *} p<0.001$. Figure is available in color online only.

\section{Results}

\section{GB and GS Group Categorization}

Of the 39 study patients ( 27 males, 12 females; mean age $63.487 \pm 9.049$ years, range $47-78$ years), 18 were categorized into the GB group and 21 into the GS group based on the radiological score and 19 into the GB group and 20 into the GS group according to quantitative cluster analysis. Discrepancies between the radiological visual analysis and quantitative cluster analysis occurred in only 3 patients and did not affect significance in further groupbased data analysis. We chose to report further analysis results using the automatic, less-subjective group categorization from the quantitative cluster analysis.

\section{Epidemiological Characteristics and Motor Function Score}

Patient sex (male/female ratio: GB 11:8, GS 20:4; $\mathrm{p}=$ 0.176 ) and disease duration (9.947 vs 9.1 years, respectively; $\mathrm{p}=0.575$ ) did not show a significant difference between the two groups. There was a statistically significantly higher average age in the GB group (66.474 years) than in the GS group (60.65 years; $p=0.0429)$. There was a significantly higher average presurgical off-medication UPDRS-III score for the GB group (51.289) than for the GS group (38.5; $\mathrm{p}=0.0372$ ). We also noted a trend for higher average on-medication UPDRS-III score for the GB group (14.421) than for the GS group (13.175), although the difference between the two groups was not statistically significant ( $p=0.671$; Fig. 2). 


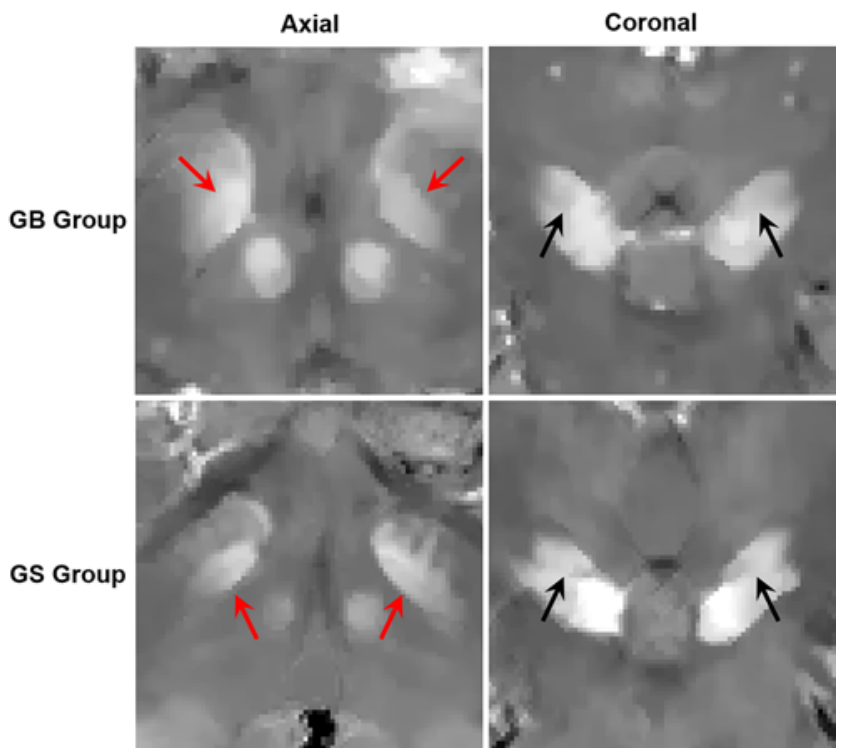

FIG. 3. Example STNs of the GB and GS groups. A subject in the GB group showed asymmetrical features of the bilateral STNs. The right STN showed a blurred boundary and a clear subdivision signal gradient. However, the left STN showed a clear boundary and a blurry subdivision signal gradient within the STN. The GS group QSM showed a clear boundary and subdivision signal gradient in both the left and right STNs. Red arrows indicate the boundary between the STN and adjacent WM; black arrows, the boundary between the STN and SN. Figure is available in color online only.

\section{Radiological Visual Results}

Figures 1 and 3 show representative examples of the STN of both the GB and GS groups in the axial and coronal QSM planes. The interrater reliability showed substantial agreement (kappa coefficient $=0.668, \mathrm{p}<0.001)$. The boundary gradient radiological score of the left STN in the GB group $(0.553 \pm 0.497)$ was significantly lower than that in the GS group ( $1 \pm 0 ; p<0.001$; Fig. 2). Similar results were observed in the right STN $(0.421 \pm 0.449$ vs 0.975 \pm 0.112 , respectively; $\mathrm{p}<0.001)$. The subdivision gradient qualitative score of the left STN in the GB group $(0.526$ $\pm 0.456)$ was also significantly lower than that in the GS group ( $1 \pm 0 ; p<0.001)$. Similar results were again observed for the right STN $(0.605 \pm 0.394$ vs $0.975 \pm 0.112$, respectively; p < 0.001; Fig. 2).

\section{Quantitative Value Results}

Between the GB and GS groups, there were no statistically significant differences in the volume of either the left STN $(\mathrm{p}=0.076)$ or right STN $(\mathrm{p}=0.846)$. There was a significantly higher QSM value for the entire STN for the GB group than the GS group, both in the left STN $(p<0.001)$ and the right STN ( $p<0.001)$. Similarly, the GB group had a significantly higher QSM value for all three subdivisions, that is, the limbic (left STN: p < 0.001, right STN: $p$ $=0.009)$, associative $(\mathrm{p}<0.001$ and $\mathrm{p}<0.001$, respectively), and motor ( $p<0.001$ and $p<0.001$, respectively) regions. The GB group also had a significantly higher QSM value for the adjacent WM than the GS group (left STN: $p$ $=0.003$, right STN: $p=0.011$; Table 1 and Fig. 4).

The GB group demonstrated a significantly higher boundary gradient in the right STN $(\mathrm{p}=0.01)$ and a trend for higher boundary gradients in the left STN $(p=0.058)$. The GB group demonstrated a significantly lower limbicassociative gradient in both the left STN $(\mathrm{p}<0.001)$ and the right STN ( $p=0.0138)$. However, there was no significant difference between the GB and GS groups in the associative-motor gradient of the left STN $(\mathrm{p}=0.116)$ and the right side STN ( $p=0.508$; Table 1 and Fig. 4).

There were significant correlations between the presurgical off-medication UPDRS-III score and STN-WM gradients $(r=0.348, p=0.002)$ and associative-motor gradients $(r=-0.294, p=0.009)$, but not with limbic-associative gradients $(\mathrm{r}=-0.103, \mathrm{p}=0.382)$.

\section{Discussion}

Our data demonstrated that both the boundary gradient and the subdivision gradient of QSM in the STN are associated with the degree of the motor function impairment in PD patients. Higher QSM values for the entire STN, the adjacent WM, and the three subdivisions of the STN were also demonstrated in the GB group, which had a higher

TABLE 1. QSM quantitative value for the STN in the GB and GS groups

\begin{tabular}{|c|c|c|c|c|c|c|}
\hline & \multicolumn{3}{|c|}{ Left STN } & \multicolumn{3}{|c|}{ Right STN } \\
\hline & GB Group & GS Group & p Value & GB Group & GS Group & $p$ Value \\
\hline $\operatorname{Vol}\left(\mathrm{mm}^{3}\right)$ & $95.553 \pm 31.892$ & $111.359 \pm 21.379$ & 0.076 & $109.994 \pm 34.236$ & $111.817 \pm 23.368$ & 0.846 \\
\hline Entire STN (ppb) & $100.354 \pm 25.344$ & $73.725 \pm 14.790$ & $<0.001$ & $98.109 \pm 24.333$ & $74.582 \pm 13.145$ & $<0.001$ \\
\hline Adjacent WM (ppb) & $19.109 \pm 19.242$ & $1.684 \pm 14.777$ & 0.003 & $16.599 \pm 16.599$ & $5.292 \pm 8.758$ & 0.011 \\
\hline Limbic region (ppb) & $136.729 \pm 29.978$ & $99.878 \pm 23.675$ & $<0.001$ & $139.286 \pm 32.587$ & $115.238 \pm 20.467$ & 0.009 \\
\hline Associative region (ppb) & $121.923 \pm 28.882$ & $70.326 \pm 26.336$ & $<0.001$ & $126.972 \pm 25.494$ & $83.802 \pm 26.934$ & $<0.001$ \\
\hline Motor region (ppb) & $59.287 \pm 33.659$ & $16.889 \pm 19.367$ & $<0.001$ & $68.155 \pm 26.391$ & $21.100 \pm 16.509$ & $<0.001$ \\
\hline STN-WM (ppb) & $81.245 \pm 18.111$ & $72.041 \pm 10.509$ & 0.058 & $81.521 \pm 15.359$ & $69.290 \pm 12.667$ & 0.01 \\
\hline L-A (ppb) & $13.763 \pm 14.185$ & $29.552 \pm 12.659$ & $<0.001$ & $12.314 \pm 24.495$ & $31.435 \pm 21.678$ & 0.014 \\
\hline A-M (ppb) & $62.636 \pm 18.453$ & $53.437 \pm 17.238$ & 0.116 & $58.931 \pm 17.490$ & $62.702 \pm 17.745$ & 0.508 \\
\hline
\end{tabular}

$\mathrm{ppb}=$ parts per billion. 

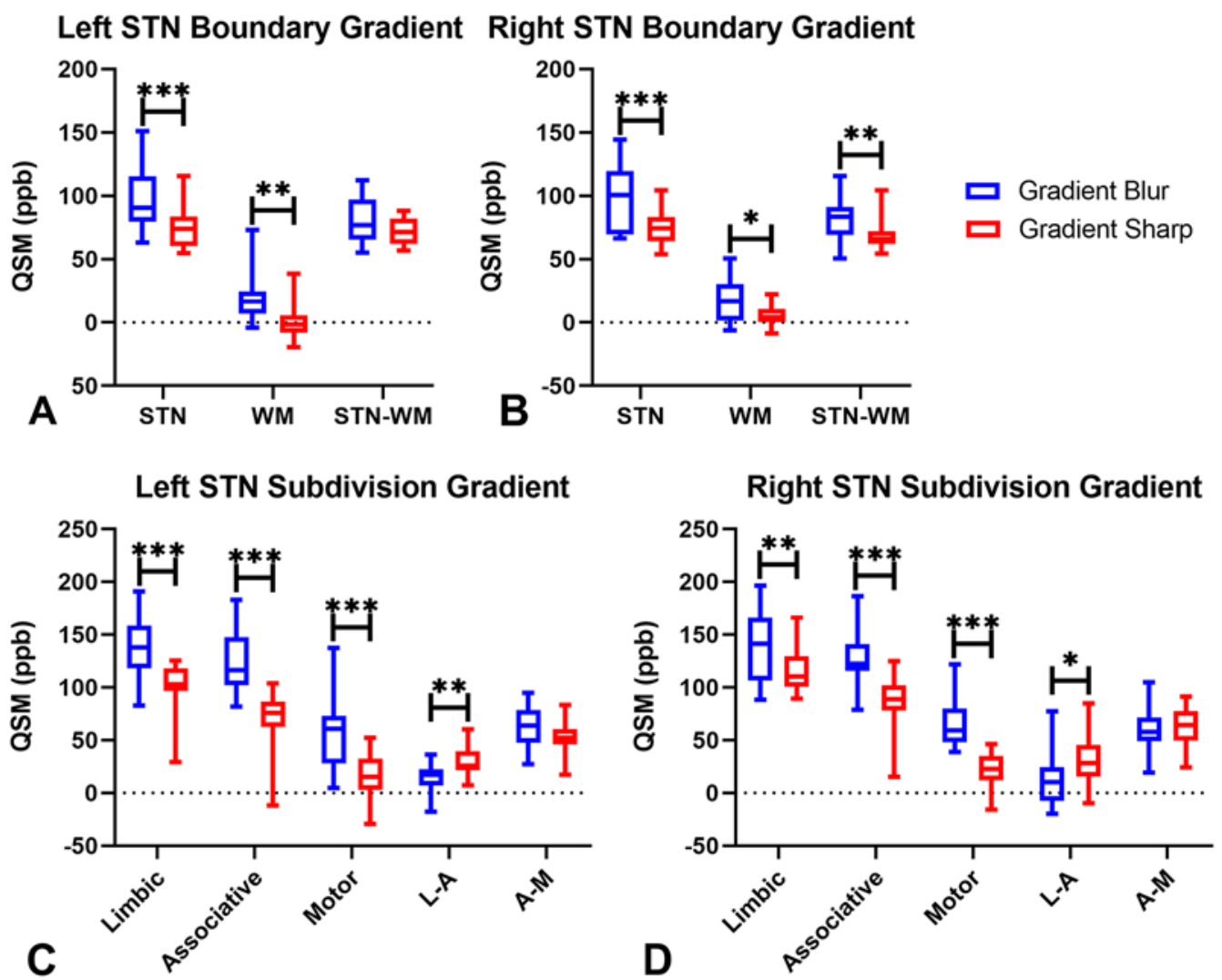

FIG. 4. QSM gradient values for the two categories of STN and its subregions and adjacent WM in the unit of parts per billion (ppb). The GB group had a significantly higher QSM value for the bilateral STNs and adjacent WM (A and B). The GB group showed a large boundary gradient (STN-WM) on the right side (B). Higher QSM values for the three subdivisions of the bilateral STNs were also observed in the GB group than in the GS group (C and D). The GB group showed a lower subdivision gradient $(\mathrm{L}-\mathrm{A})$ in the bilateral STNs. ${ }^{*} p<0.05,{ }^{* *} p<0.01$, and ${ }^{* * *} p<0.001$. Figure is available in color online only.

average presurgical off-medication UPDRS-III score. A decrease in the limbic-associative subdivision gradient was observed in the GB group.

Several histological and radiological studies have reported an increased iron concentration in the deep nuclei of patients with PD, especially the SN. ${ }^{19-21}$ QSM allows for the quantification of tissue iron content and could be sensitive to microstructural abnormalities in neurodegenerative disease. ${ }^{22}$ The STN is a key nucleus in the functional control of motor activity in the basal ganglia by receiving GABAergic inhibitory projections from the globus pallidus externa (GPe). Patients with PD have a hyperactive STN because of the loss of GABAergic inhibitory projections from the GPe, but STN neurons continue to receive the excitatory drive of the cortex. This may lead to excitotoxic injury of the STN neurons, excessive generation of free radicals, and increased iron deposition within the $\mathrm{STN},{ }^{23}$ which can be noninvasively quantitatively measured using QSM.

Furthermore, increased iron content in the $\mathrm{SN}, \mathrm{RN}$, and globus pallidus and its correlation with disease severity has been demonstrated. ${ }^{24,25}$ Kosta et al. ${ }^{23}$ first reported that iron deposition was increased in the STN in PD patients with a disease duration $>5$ years. However, these authors did not evaluate the correlation between STN iron deposition and motor impairment. Our results revealed that the
GB group has higher UPDRS-III scores and higher QSM values for the entire STN and adjacent WM. Advancing PD with more severe motor impairment leads to more iron deposition in the STN, which is in agreement with previous studies of other nuclei. ${ }^{24}$ The many anatomical and functional interconnections of these deep nuclei may contribute to these changes. Our finding that PD patients with worse motor function impairment had more iron deposition in the near WM of the STN has not been reported in the literature. This finding could explain why a more indistinct boundary of the STN was observed in patients with more motor impairment. However, the STN-WM iron difference was greater in the GB group than in the GS group. A possible interpretation is that the excessive pathological iron deposition contributing to motor function impairment is centered in the STN but diffuses into the adjacent space, resulting in an iron contrast increase but an iron boundary blur between the STN and adjacent WM.

Previous findings have confirmed heterogeneous iron distributions throughout the STN both in vivo and postmortem. ${ }^{26,27}$ Furthermore, de Hollander et al. ${ }^{9}$ demonstrated increased iron concentrations along the medialinferior-anterior direction in the STN. We found that all three subdivisions of the STN in the GB group have more iron deposition than those in the GS group. Among the 
three subdivisions, the iron concentration of the limbic region and the associative region was greater than that of the motor region. This distribution is consistent with previous research. ${ }^{9}$ The GB group showed less of an interior gradient than the GS group, especially within the limbicassociative region gradient. One possible interpretation is that abnormal iron deposition in the STN in the GB group diffuses within the STN subregions, leading to a blurry signal gradient among the three subdivisions. Iron deposition tends to occur mostly in the associative region, leading to a decreased L-A gradient. The loss of the STN inner QSM signal gradient tends to be associated with higher UPDRS-III scores, indicating more severe motor impairment according to data in this study. Because of the anatomical and functional overlapping of STN subdivisions, the degree to which iron deposition in any particular functional subdivision of the STN correlates with the degree of motor function impairment must be investigated in further studies.

PD patients mainly present with motor impairment symptoms, and our results confirmed that greater motor function impairment tends to occur with greater iron deposition within the STN and the boundary area, particularly in the associative subregion. Loss of the STN QSM signal gradient should be considered as an image marker for more severe motor impairment in PD patients.

The present study has the following limitations. First, the analyses in this work were performed on a relatively small number of patients. Second, both anatomical and functional subdivisions of the STN overlapped with each other-it is difficult to distinguish the three areas precisely. Therefore, we subdivided the STN into three equal parts from ventromedial to dorsolateral, representing three functional regions. More precise separation should be used in future studies. Finally, no DBS response or clinical followup was included in the current analyses. Given our demonstration of iron gradient relationship to motoric impairment, a natural next step would be to determine the clinical response to different gradient types in further studies.

\section{Conclusions}

We demonstrate for the first time that the boundary and subdivision QSM signal gradient of the STN on MRI correlates with motor impairment in PD patients and may aid in the optimization of patient selection for DBS treatment.

\section{References}

1. Calabresi P, Picconi B, Tozzi A, et al. Direct and indirect pathways of basal ganglia: a critical reappraisal. Nat Neurosci. 2014;17(8):1022-1030.

2. Obeso I, Wilkinson L, Rodríguez-Oroz MC, et al. Bilateral stimulation of the subthalamic nucleus has differential effects on reactive and proactive inhibition and conflict-induced slowing in Parkinson's disease. Exp Brain Res. 2013;226(3): 451-462.

3. Alkemade A, Schnitzler A, Forstmann BU. Topographic organization of the human and non-human primate subthalamic nucleus. Brain Struct Funct. 2015;220(6):3075-3086.

4. Weaver F, Follett K, Hur K, et al. Deep brain stimulation in Parkinson disease: a metaanalysis of patient outcomes. $J$ Neurosurg. 2005;103(6):956-967.

5. Wong JK, Cauraugh JH, Ho KWD, et al. STN vs. GPi deep brain stimulation for tremor suppression in Parkinson disease: a systematic review and meta-analysis. Parkinsonism Relat Disord. 2019;58:56-62.

6. de Rochefort L, Liu T, Kressler B, et al. Quantitative susceptibility map reconstruction from MR phase data using bayesian regularization: validation and application to brain imaging. Magn Reson Med. 2010;63(1):194-206.

7. Liu T, Eskreis-Winkler S, Schweitzer AD, et al. Improved subthalamic nucleus depiction with quantitative susceptibility mapping. Radiology. 2013;269(1):216-223.

8. Dimov AV, Gupta A, Kopell BH, Wang Y. High-resolution QSM for functional and structural depiction of subthalamic nuclei in DBS presurgical mapping. J Neurosurg. 2019;131(2): 360-367.

9. de Hollander G, Keuken MC, Bazin PL, et al. A gradual increase of iron toward the medial-inferior tip of the subthalamic nucleus. Hum Brain Mapp. 2014;35(9):4440-4449.

10. Karachi C, Yelnik J, Tandé D, et al. The pallidosubthalamic projection: an anatomical substrate for nonmotor functions of the subthalamic nucleus in primates. Mov Disord. 2005; 20(2):172-180.

11. Lambert C, Zrinzo L, Nagy Z, et al. Confirmation of functional zones within the human subthalamic nucleus: patterns of connectivity and sub-parcellation using diffusion weighted imaging. Neuroimage. 2012;60(1):83-94.

12. Dimov A, Patel W, Yao Y, et al. Iron concentration linked to structural connectivity in the subthalamic nucleus: implications for deep brain stimulation. J Neurosurg. 2020;132(1): 197-204.

13. Fahn S, Marsden CD, Calne DB, Goldstein M, eds. Recent Development in Parkinson's Disease. Macmillan Health Care Information; 1987:153-164.

14. Liu Z, Kee Y, Zhou D, et al. Preconditioned total field inversion (TFI) method for quantitative susceptibility mapping. Magn Reson Med. 2017;78(1):303-315.

15. Liu Z, Spincemaille P, Yao Y, et al. MEDI+0: morphology enabled dipole inversion with automatic uniform cerebrospinal fluid zero reference for quantitative susceptibility mapping. Magn Reson Med. 2018;79(5):2795-2803.

16. Spincemaille $\mathrm{P}$, Liu Z, Zhang S, et al. Clinical integration of automated processing for brain quantitative susceptibility mapping: multi-site reproducibility and single-site robustness. J Neuroimaging. 2019;29(6):689-698.

17. Yushkevich PA, Piven J, Hazlett HC, et al. User-guided 3D active contour segmentation of anatomical structures: significantly improved efficiency and reliability. Neuroimage. 2006; 31(3):1116-1128.

18. Arthur D, Vassilvitskii S. Siam/Acm, k-means plus plus: the advantages of careful seeding. In: Proceedings of the Eighteenth Annual ACM-SIAM Symposium on Discrete Algorithms. IEEE; 2007:1027-1035.

19. Sofic E, Riederer P, Heinsen H, et al. Increased iron (III) and total iron content in post mortem substantia nigra of parkinsonian brain. J Neural Transm (Vienna). 1988;74(3):199-205.

20. Virel A, Faergemann E, Orädd G, Strömberg I. Magnetic resonance imaging (MRI) to study striatal iron accumulation in a rat model of Parkinson's disease. PLoS One. 2014;9(11): e112941.

21. Martin-Bastida A, Politis M, Loane C, et al. Susceptibility weighted imaging to detect nigral iron accumulation in Parkinson's disease. J Neurol Neurosurg Psychiatry. 2015;86:e4.

22. Mazzucchi S, Frosini D, Costagli M, et al. Quantitative susceptibility mapping in atypical Parkinsonisms. Neuroimage Clin. 2019;24:101999.

23. Kosta P, Argyropoulou MI, Markoula S, Konitsiotis S. MRI evaluation of the basal ganglia size and iron content in patients with Parkinson's disease. J Neurol. 2006;253(1):26-32.

24. Shahmaei V, Faeghi F, Mohammdbeigi A, et al. Evaluation of iron deposition in brain basal ganglia of patients with Parkin- 
son's disease using quantitative susceptibility mapping. Eur J Radiol Open. 2019;6:169-174.

25. Jin L, Wang J, Jin H, et al. Nigral iron deposition occurs across motor phenotypes of Parkinson's disease. Eur J Neurol. 2012;19(7):969-976.

26. Massey LA, Miranda MA, Zrinzo L, et al. High resolution MR anatomy of the subthalamic nucleus: imaging at $9.4 \mathrm{~T}$ with histological validation. Neuroimage. 2012;59(3):20352044.

27. Dormont D, Ricciardi KG, Tandé D, et al. Is the subthalamic nucleus hypointense on T2-weighted images? A correlation study using MR imaging and stereotactic atlas data. AJNR Am J Neuroradiol. 2004;25(9):1516-1523.

\section{Disclosures}

Dr. Wang has direct stock ownership in Medimagemetric LLC.
Dr. Kopell has been a consultant for Medtronic, Abbott, and Elekta.

\section{Author Contributions}

Conception and design: Kopell, Wang. Acquisition of data: Kopell, Ogbuji. Analysis and interpretation of data: Huang, Zhou, Guo, Wang. Drafting the article: Huang, Ogbuji. Critically revising the article: Kopell, Ogbuji, Wang. Reviewed submitted version of manuscript: all authors. Approved the final version of the manuscript on behalf of all authors: Kopell. Statistical analysis: Zhou. Administrative/technical/material support: Kopell. Study supervision: Kopell, Wang.

\section{Correspondence}

Brian H. Kopell: Icahn School of Medicine at Mount Sinai, New York, NY.brian.kopell@mountsinai.org. 